\title{
Convergence of Ishikawa iterates of two mappings in modular function spaces
}

\author{
Afrah Ahmad Noan Abdou ${ }^{1 *}$, Mohamed Amine Khamsi2,3 and Abdul Rahim Khan ${ }^{3}$
}

"Correspondence:

aabdou@kau.edu.sa

${ }^{1}$ Department of Mathematics, King Abdulaziz University, Jeddah, 21589,

Saudi Arabia

Full list of author information is

available at the end of the article

\begin{abstract}
We establish convergence in the modular sense of an iteration scheme associated with a pair of mappings on a nonlinear domain in modular function spaces. In particular, we prove that such a scheme converges to a common fixed point of the mappings. Our results are generalization of known similar results in the non-modular setting. In particular, we avoid smoothness of the norm in the case of Banach spaces and that of the triangle inequality of the distance in metric spaces.
\end{abstract}

MSC: Primary 47H09; secondary 46B20; 47H10; 47E10

Keywords: fixed point; fixed point iteration process; Ishikawa iterations; modular function space; nonexpansive mapping; $\rho$-convergence; modular function space; Orlicz space; strict convexity

\section{Introduction and basic definitions}

The earliest attempts to generalize the classical function spaces $L^{p}$ of Lebesgue type were made in the early 1930s by Orlicz and Birnbaum in connection with orthogonal expansions. Their approach consisted in considering spaces of functions with some growth properties different from the power type growth control provided by the $L^{p}$-norms. Namely, they considered the function spaces defined as follows:

$$
L^{\varphi}=\left\{f: \mathbb{R} \rightarrow \mathbb{R} ; \exists \lambda>0: \int_{\mathbb{R}} \varphi(\lambda|f(x)|) d x<\infty\right\},
$$

where $\varphi:[0, \infty] \rightarrow[0, \infty]$ was assumed to be a convex function increasing to infinity, i.e. the function which to some extent behaves similar to power functions $\varphi(t)=t^{p}$. Later on, the assumption of convexity for Orlicz functions $\varphi$ was frequently omitted. Let us mention two typical examples of such functions:

$$
\varphi(t)=e^{t}-1, \quad \varphi(t)=\ln (1+t)
$$

The possibility of introducing the structure of a linear metric in $L^{\varphi}$ as well as the interesting properties of these spaces and many applications to differential and integral equations with kernels of nonpower types were among the reasons for the development of the theory of Orlicz spaces, their applications, and generalizations for more than half a century.

We may observe two principal directions of further development. The first is the theory of Banach function spaces initiated in 1955 by Luxemburg [1] and developed further

\section{Springer}

@2014 Abdou et al.; licensee Springer. This is an Open Access article distributed under the terms of the Creative Commons Attribution License (http://creativecommons.org/licenses/by/2.0), which permits unrestricted use, distribution, and reproduction in any medium, provided the original work is properly cited. 
in a series of joint papers with Zaanen [2]. The second one is inspired by the theory of Orlicz spaces, based on replacing the particular integral form of the nonlinear functional, which controls the growth of members of the space, by an abstract given functional with some good properties. This idea was the basis of the theory of modular spaces initiated by Nakano [3] in connection with the theory of order spaces and redefined and generalized by Luxemburg and Orlicz in 1959. Such spaces have been studied for almost 40 years and a number of applications of such spaces in various parts of analysis, probability, and mathematical statistics are known.

In this paper, we will consider modular spaces which lie somewhere in between the abstract modular theory and Musielak-Orlicz theory, i.e. the class of modular spaces given by modulars not of any particular form but, nevertheless, having much more convenient properties than the abstract modulars can possess. In other words, we present a useful tool for applications whenever there is a need to introduce a function space by means of functionals which have some reasonable properties but are far from being norms or $F$-norms.

Let us introduce basic notions in modular function spaces and related notations which will be used in this chapter. For further details we refer the reader to preliminary sections of the recent articles [4-6] or to the survey article [7]; see also [8-10] for the standard framework of modular function spaces.

Let $\Omega$ be a nonempty set and $\Sigma$ be a nontrivial $\sigma$-algebra of subsets of $\Omega$. Let $\mathcal{P}$ be a $\delta$-ring of subsets of $\Omega$, such that $E \cap A \in \mathcal{P}$ for any $E \in \mathcal{P}$ and $A \in \Sigma$. Let us assume that there exists an increasing sequence of sets $K_{n} \in \mathcal{P}$ such that $\Omega=\bigcup K_{n}$. By $\mathcal{E}$ we denote the linear space of all simple functions with support from $\mathcal{P}$. By $\mathcal{M}_{\infty}$ we will denote the space of all extended measurable functions, i.e. all functions $f: \Omega \rightarrow[-\infty, \infty]$ such that there exists a sequence $\left\{g_{n}\right\} \subset \mathcal{E},\left|g_{n}\right| \leq|f|$ and $g_{n}(\omega) \rightarrow f(\omega)$ for all $\omega \in \Omega$. By $1_{A}$ we denote the characteristic function of the set $A$.

Definition 1.1 [10] Let $\rho: \mathcal{M}_{\infty} \rightarrow[0, \infty]$ be a nontrivial, convex, and even function. We say that $\rho$ is a regular convex function pseudomodular if:

(i) $\rho(0)=0$;

(ii) $\rho$ is monotone, i.e. $|f(\omega)| \leq|g(\omega)|$ for all $\omega \in \Omega$ implies $\rho(f) \leq \rho(g)$, where $f, g \in \mathcal{M}_{\infty}$;

(iii) $\rho$ is orthogonally subadditive, i.e. $\rho\left(f 1_{A \cup B}\right) \leq \rho\left(f 1_{A}\right)+\rho\left(f 1_{B}\right)$ for any $A, B \in \Sigma$ such that $A \cap B \neq \emptyset, f \in \mathcal{M}$;

(iv) $\rho$ has the Fatou property, i.e. $\left|f_{n}(\omega)\right| \uparrow|f(\omega)|$ for all $\omega \in \Omega$ implies $\rho\left(f_{n}\right) \uparrow \rho(f)$, where $f \in \mathcal{M}_{\infty}$;

(v) $\rho$ is order continuous in $\mathcal{E}$, i.e. $g_{n} \in \mathcal{E}$ and $\left|g_{n}(\omega)\right| \downarrow 0$ implies $\rho\left(g_{n}\right) \downarrow 0$.

Similarly, as in the case of measure spaces, we say that a set $A \in \Sigma$ is $\rho$-null if $\rho\left(g 1_{A}\right)=0$ for every $g \in \mathcal{E}$. We say that a property holds $\rho$-almost everywhere if the exceptional set is $\rho$-null. As usual we identify any pair of measurable sets whose symmetric difference is $\rho$-null as well as any pair of measurable functions differing only on a $\rho$-null set. With this in mind, we define $\mathcal{M}=\left\{f \in \mathcal{M}_{\infty} ;|f(\omega)|<\infty \rho\right.$-a.e. $\}$, where each element is actually an equivalence class of functions equal $\rho$-a.e. rather than an individual function.

Definition 1.2 [10] We say that a regular function pseudomodular $\rho$ is a regular convex function modular if $\rho(f)=0$ implies $f=0 \rho$-a.e. The class of all nonzero regular convex function modulars defined on $\Omega$ will be denoted by $\Re$. 
In this paper, we only consider convex function modulars.

Definition 1.3 [8-10] Let $\rho$ be a convex function modular. A modular function space is the vector space $L_{\rho}=\{f \in \mathcal{M} ; \rho(\lambda f) \rightarrow 0$ as $\lambda \rightarrow 0\}$. In the vector space $L_{\rho}$, the following formula:

$$
\|f\|_{\rho}=\inf \left\{\alpha>0 ; \rho\left(\frac{f}{\alpha}\right) \leq 1\right\}
$$

defines a norm, frequently called the Luxemburg norm.

Note that the monographic exposition of the theory of Orlicz spaces may be found in the book of Krasnosel'skii and Rutickii [11]. For a current review of the theory of Musielak-Orlicz spaces and modular spaces, the reader is referred to Musielak [12] and Kozlowski [10].

The following definitions will be needed in this paper.

\section{Definition $1.4[10]$}

(a) The sequence $\left\{f_{n}\right\} \subset L_{\rho}$ is said to be $\rho$-convergent to $f \in L_{\rho}$ if $\rho\left(f_{n}-f\right) \rightarrow 0$ as $n \rightarrow \infty$.

(b) The sequence $\left\{f_{n}\right\} \subset L_{\rho}$ is said to be $\rho$-Cauchy if $\rho\left(f_{n}-f_{m}\right) \rightarrow 0$ as $n$ and $m \rightarrow \infty$.

(c) We say that $L_{\rho}$ is $\rho$-complete if and only if any $\rho$-Cauchy sequence in $L_{\rho}$ is $\rho$-convergent.

(d) A subset $C$ of $L_{\rho}$ is called $\rho$-closed if the $\rho$-limit of a $\rho$-convergent sequence of $C$ always belongs to $C$.

(e) A subset $C$ of $L_{\rho}$ is called $\rho$-compact if every sequence in $C$ has a $\rho$-convergent subsequence in $C$.

(f) A subset $C$ of $L_{\rho}$ is called $\rho$-bounded if

$$
\delta_{\rho}(C)=\sup \{\rho(f-g) ; f, g \in C\}<\infty .
$$

(g) Let $f \in L_{\rho}$ and $C \subset L_{\rho}$. Define the $\rho$-distance between $f$ and $C$ as:

$$
d_{\rho}(f, C)=\inf \{\rho(f-g) ; g \in C\} .
$$

The above terminology is used because of its similarity to the metric case. Since $\rho$ does not behave in general as a distance, one should be very careful when dealing with these notions. In particular, $\rho$-convergence does not imply $\rho$-Cauchy since $\rho$ does not satisfy the triangle inequality.

The following proposition brings together a few facts that will be often used.

Proposition 1.1 [10] Let $\rho \in \Re$.

(i) $L_{\rho}$ is $\rho$-complete.

(ii) $\rho$-balls $B_{\rho}(f, r)=\left\{g \in L_{\rho} ; \rho(f-g) \leq r\right\}$ are $\rho$-closed.

(iii) If $\rho\left(\alpha f_{n}\right) \rightarrow 0$ for an $\alpha>0$ then there exists a subsequence $\left\{g_{n}\right\}$ of $\left\{f_{n}\right\}$ such that $g_{n} \rightarrow 0 \rho$-a.e.

(iv) $\rho(f) \leq \liminf _{n \rightarrow \infty} \rho\left(f_{n}\right)$ whenever $f_{n} \rightarrow f \rho$-a.e. (Note: this property is equivalent to the Fatou property.) 
(v) Consider the sets $L_{\rho}^{0}=\left\{f \in L_{\rho} ; \rho(f, \cdot)\right.$ is order continuous $\}$, and $E_{\rho}=\left\{f \in L_{\rho} ; \lambda f \in L_{\rho}^{0}\right.$ for any $\left.\lambda>0\right\}$. Then we have $E_{\rho} \subset L_{\rho}^{0} \subset L_{\rho}$.

We already pointed out that $\rho$ may not satisfy the triangle inequality, so the modular convergence and norm convergence may not be the same. This will only happen if $\rho$ satisfies the so-called $\Delta_{2}$-condition.

Definition 1.5 [10] The modular function $\rho$ is said to satisfy the $\Delta_{2}$-condition if $\rho\left(2 f_{n}\right) \rightarrow$ 0 as $n \rightarrow \infty$, whenever $\rho\left(f_{n}\right) \rightarrow 0$ as $n \rightarrow \infty$.

We have the following proposition.

Proposition 1.2 [10] The following statements are equivalent:

(i) $\rho$ satisfies the $\Delta_{2}$-condition;

(ii) $\rho\left(f_{n}-f\right) \rightarrow 0$ if and only if $\rho\left(\lambda\left(f_{n}-f\right)\right) \rightarrow 0$, for all $\lambda>0$ if and only if $\left\|f_{n}-f\right\| \rightarrow 0$.

Definition 1.6 We will say that the function modular $\rho$ is uniformly continuous if for every $\varepsilon>0$ and $L>0$, there exists $\delta>0$ such that

$$
|\rho(g)-\rho(h+g)|<\varepsilon ; \quad \text { if } \rho(h)<\delta \text { and } \rho(g) \leq L .
$$

Let us mention that uniform continuity holds for a large class of function modulars. For instance, it can be proved that in Orlicz spaces over a finite atomless measure [13] or in sequence Orlicz spaces [14], the uniform continuity of the Orlicz modular is equivalent to the $\Delta_{2}$-type condition.

Let us recall the definition of different mappings acting in a modular function space. We start with the concept of Lipschitzian mappings.

Definition 1.7 Let $\rho \in \mathfrak{R}$ and let $C \subset L_{\rho}$ be a nonempty subset. A mapping $T: C \rightarrow C$ is called $\rho$-Lipschitzian mapping if there exists a constant $L \geq 0$ such that

$$
\rho(T(f)-T(g)) \leq L \rho(f-g) \text { for any } f, g \in C \text {. }
$$

When $L<1, T$ is called $\rho$-contraction mapping. Moreover, if $L \leq 1$, then $T$ is called $\rho$-nonexpansive mapping. A point $f \in C$ is called a fixed point of $T$ if $T(f)=f$. The set of fixed points of $T$ will be denoted by $F(T)$.

As mentioned before, one of the reasons of our interest in $\rho$-behavior of mappings is that the Luxemburg norm associated with the function modular is defined in an indirect way and consequently harder to handle than the function modular. Therefore, one may ask what the relationship is, if there is any, between the $F$-norm nonexpansiveness and the $\rho$-nonexpansiveness. The following example gives a partial answer.

Example 1.1 [15] Let $X=(0, \infty)$, and let $\Sigma$ be the $\sigma$-algebra of all Lebesgue measurable subsets of $X$. Let $\mathcal{P}$ denote the $\delta$-ring of subsets of finite measures. Define a function modular by

$$
\rho(f)=\frac{1}{e^{2}} \int_{0}^{\infty}|f(x)|^{x+1} d m(x) .
$$


Let $B$ be the set of all measurable functions $f:(0, \infty) \rightarrow \mathbb{R}$ such that $0 \leq f(x) \leq \frac{1}{2}$. Define the linear operator $T$ by the formula

$$
T(f)(x)= \begin{cases}f(x-1) & \text { for } x \geq 1 \\ 0 & \text { for } x \in[0,1]\end{cases}
$$

Clearly, $T(B) \subset B$. In [15], it is proved that for every fixed $\lambda \leq 1$ and for all $f, g \in B$, we have

$$
\rho(\lambda(T(f)-T(g))) \leq \lambda \rho(\lambda(f-g))
$$

In particular, if $\lambda=1$, then the above inequality shows that $T$ is $\rho$-nonexpansive. It is also easy to see that $B$ is a $\rho$-a.e.-bounded subset of $L_{\rho}$. We observe that $T$ is not $\|\cdot\|_{\rho}$ nonexpansive. Indeed, if we take $f=1_{[0,1]}$, then

$$
\|T(f)\|_{\rho}>e \geq\|f\|_{\rho} .
$$

In this paper, we will use some geometrical properties of the modular functional.

Definition 1.8 Let $\rho \in \mathfrak{R}$. We say that $\rho$ is strictly convex (SC), if for every $f, g \in L_{\rho}$ such that $\rho(f)=\rho(g)$ and $\rho(\alpha f+(1-\alpha) g)=\alpha \rho(f)+(1-\alpha) \rho(g)$, for some $\alpha \in(0,1)$, we have $f=g$.

It is known that for a wide class of modular function spaces with the $\Delta_{2}$-property, geometric properties of the Luxemburg norm are equivalent to the same properties of the modular. For example, in Orlicz spaces these results can be traced in early papers of Luxemburg [1], Milnes [16], Akimovic [17], and Kaminska [14]. It is also known that, under suitable assumptions, uniform convexity in Orlicz spaces is equivalent to the very convexity of the Orlicz function [18]. Typical examples of Orlicz functions that do not satisfy the $\Delta_{2}$-condition but are uniformly convex (and hence strictly convex) are $[11,16]$

$$
\varphi_{1}(t)=e^{|t|}-|t|-1 \quad \text { and } \quad \varphi_{2}(t)=e^{t^{2}}-1
$$

For the discussion of some geometrical properties of Calderon-Lozanovskii and OrliczLorentz spaces, the reader may consult [19].

Recently, special attention was given to the use of the geometric properties in modular function spaces. This is due to recent interest in the Dirichlet energy problem which we discuss in the next example.

Example 1.2 Let $\Omega \subset \mathbb{R}$ be an open set and let $p: \Omega \rightarrow[1, \infty)$ be a measurable function (called the variable exponent on $\Omega$ ). We define the variable exponent Lebesgue space $L^{p(\cdot)}(\Omega)$ to consist of all measurable functions $f: \Omega \rightarrow \mathbb{R}$ such that

$$
\rho(\lambda f)=\int_{\Omega}|\lambda f(x)|^{p(x)} d x<\infty
$$

for some $\lambda>0$. The functional $\rho$ is called the modular of the space $L^{p(\cdot)}(\Omega)$. The Luxemburg norm on this space is given by the formula

$$
\|f\|=\inf \{\lambda>0 ; \rho(\lambda f)<\infty\}
$$


The variable exponent Sobolev space $W^{1, p(\cdot)}(\Omega)$ is the space of measurable functions $f$ : $\Omega \rightarrow \mathbb{R}$ such that $f$ and the distributional derivative $f^{\prime}$ are in $L^{p(\cdot)}(\Omega)$. The function

$$
\rho_{1}(f)=\rho(f)+\rho\left(f^{\prime}\right)
$$

defines a modular on $W^{1, p(\cdot)}(\Omega)$. Define $W_{0}^{1, p(\cdot)}(\Omega)$ as the set of $f \in W^{1, p(\cdot)}(\Omega)$ which can be continuously continued by 0 outside $\Omega$. The energy operator corresponding to the boundary value function $g$ acting on the space

$$
\left\{f \in W^{1, p(\cdot)}(\Omega) ; f-g \in W_{0}^{1, p(\cdot)}(\Omega)\right\}
$$

is defined by

$$
I_{g}(f)=\int_{\Omega}\left|f^{\prime}(x)\right|^{p(x)} d x=\rho\left(f^{\prime}\right) .
$$

The general Dirichlet energy problem is to find a function that minimizes values of the operator $I_{g}(\cdot)$. Note that

$$
\min \left\{I_{g}(g-f) ; f \in W_{0}^{1, p(\cdot)}(\Omega)\right\}=d_{\rho}\left(g, W_{0}^{1, p(\cdot)}(\Omega)\right) .
$$

For more information on the Dirichlet energy integral problem we refer to [20-23].

\section{Ishikawa iterates for two mappings}

In [24], the authors introduced the Ishikawa iterative scheme for two mappings and studied the strong convergence of this scheme to a common fixed point of the two mappings. Let us introduce such an iterative scheme in modular function spaces. Let $S$ and $T$ be two mappings defined on a nonempty closed, convex and $\rho$-bounded subset $C$ of $L_{\rho}$. Fix $f \in C$ and define the sequence $\left\{f_{n}\right\}$, with $f_{1}=f$, and

$$
f_{n+1}=\alpha_{n} S\left(\beta_{n} T\left(f_{n}\right)+\left(1-\beta_{n}\right) f_{n}\right)+\left(1-\alpha_{n}\right) f_{n}, \quad n=1,2, \ldots
$$

When $S=T$, the above iterative scheme collapses into the classical Ishikawa iterative scheme for one map:

$$
f_{n+1}=\alpha_{n} T\left(\beta_{n} T\left(f_{n}\right)+\left(1-\beta_{n}\right) f_{n}\right)+\left(1-\alpha_{n}\right) f_{n}, \quad n=1,2, \ldots
$$

Let $S, T: C \rightarrow C$ be two $\rho$-nonexpansive mappings. Assume that $F=F(S) \cap F(T) \neq \emptyset$. Let $f \in C$ and $h \in F$. Set $r=\rho(f-h)$. Then

$$
C(f)=C \cap B(h, r)=\{g \in C ; \rho(h-g) \leq r\}
$$

is a nonempty closed and convex subset of $C$ and invariant under both $S$ and $T$. Therefore one may always assume that $C$ is $\rho$-bounded once $S$ and $T$ have a common fixed point. Moreover, if $\left\{f_{n}\right\}$ is the sequence generated by (2.1), with $f_{1}=f$, then we have

$$
\begin{aligned}
\rho\left(f_{n+1}-h\right) & =\rho\left(\alpha_{n} S\left(g_{n}\right)+\left(1-\alpha_{n}\right) f_{n}-h\right) \\
& \leq \alpha_{n} \rho\left(S\left(g_{n}\right)-h\right)+\left(1-\alpha_{n}\right) \rho\left(f_{n}-h\right)
\end{aligned}
$$




$$
\begin{aligned}
& \leq \alpha_{n} \rho\left(g_{n}-h\right)+\left(1-\alpha_{n}\right) \rho\left(f_{n}-h\right) \\
& =\alpha_{n} \rho\left(\beta_{n} T\left(f_{n}\right)+\left(1-\beta_{n}\right) f_{n}-h\right)+\left(1-\alpha_{n}\right) \rho\left(f_{n}-h\right) \\
& \leq \alpha_{n}\left[\beta_{n} d\left(T\left(f_{n}\right)-h\right)+\left(1-\beta_{n}\right) \rho\left(f_{n}-h\right)\right]+\left(1-\alpha_{n}\right) \rho\left(f_{n}-h\right) \\
& \leq \rho\left(f_{n}-h\right)
\end{aligned}
$$

where $g_{n}=\beta_{n} T\left(f_{n}\right)+\left(1-\beta_{n}\right) f_{n}$. This proves that $\left\{\rho\left(f_{n}-h\right)\right\}$ is decreasing, which implies that $\lim _{n \rightarrow \infty} \rho\left(f_{n}-h\right)$ exists. Using the above inequalities, we get

$$
\begin{aligned}
\lim _{n \rightarrow \infty} \rho\left(f_{n}-h\right) & =\lim _{n \rightarrow \infty} \rho\left(\alpha_{n} S\left(g_{n}\right)+\left(1-\alpha_{n}\right) f_{n}-h\right) \\
& =\lim _{n \rightarrow \infty} \alpha_{n} \rho\left(S\left(g_{n}\right)-h\right)+\left(1-\alpha_{n}\right) \rho\left(f_{n}-h\right) \\
& =\lim _{n \rightarrow \infty} \alpha_{n} \rho\left(g_{n}-h\right)+\left(1-\alpha_{n}\right) \rho\left(f_{n}-h\right) \\
& =\lim _{n \rightarrow \infty} \alpha_{n} \alpha_{n} \rho\left(\beta_{n} T\left(f_{n}\right)+\left(1-\beta_{n}\right) f_{n}-h\right)+\left(1-\alpha_{n}\right) \rho\left(f_{n}-h\right) \\
& =\lim _{n \rightarrow \infty} \alpha_{n}\left[\beta_{n} d\left(T\left(f_{n}\right)-h\right)+\left(1-\beta_{n}\right) \rho\left(f_{n}-h\right)\right]+\left(1-\alpha_{n}\right) \rho\left(f_{n}-h\right) .
\end{aligned}
$$

Our first result discusses the convergence behavior of the sequence generated by (2.1).

Theorem 2.1 Assume $\rho \in \Re$ is strictly convex and uniformly continuous. Let $C$ be a nonempty $\rho$-bounded, closed and convex subset of $X$. Let $S, T: C \rightarrow C$ be two $\rho$-nonexpansive mappings. Assume that $F=F(S) \cap F(T) \neq \emptyset$. Let $f_{1} \in C$ and $\left\{f_{n}\right\}$ be given by (2.1). Then the following hold:

(i) If $\alpha_{n} \in[a, b]$ and $\beta_{n} \in[0, b]$, with $0<a \leq b<1$, then for any subsequence $\left\{f_{n_{i}}\right\}$ of $\left\{f_{n}\right\}$ which $\rho$-converges to $f$, we have $f \in F(S)$.

(ii) If $\alpha_{n} \in[a, 1]$ and $\beta_{n} \in[a, b]$, with $0<a \leq b<1$, then for any subsequence $\left\{f_{n_{i}}\right\}$ of $\left\{f_{n}\right\}$ which $\rho$-converges to $f$, we have $f \in F(T)$.

(iii) If $\alpha_{n}, \beta_{n} \in[a, b]$, with $0<a \leq b<1$, then for any subsequence $\left\{f_{n_{i}}\right\}$ of $\left\{f_{n}\right\}$ which $\rho$-converges to $f$, we have $f \in F$. In this case, we have that $\left\{f_{n}\right\} \rho$-converges to $f$.

Proof Assume that $\left\{f_{n_{i}}\right\} \rho$-converges to $f$. Let $h \in F$. Without loss of generality, we may assume $\lim _{n \rightarrow \infty} \alpha_{n_{i}}=\alpha$, and $\lim _{n \rightarrow \infty} \beta_{n_{i}}=\beta$. Since $\left\{\rho\left(f_{n}-h\right)\right\}$ is decreasing and $\rho$ is uniformly continuous, we get

$$
\lim _{n \rightarrow \infty} \rho\left(f_{n}-h\right)=\lim _{n \rightarrow \infty} \rho\left(f_{n_{i}}-h\right)=\rho(f-h) .
$$

Since $T$ is $\rho$-nonexpansive, $\left\{T\left(f_{n_{i}}\right)\right\} \rho$-converges to $T(f)$. Moreover, as $\rho$ is uniformly continuous, $\left\{\beta_{n_{i}} T\left(f_{n_{i}}\right)+\left(1-\beta_{n_{i}}\right) f_{n_{i}}\right\} \rho$-converges to $\beta T(f)+(1-\beta) f$. Using the $\rho$-nonexpansiveness of $S$, we get $\left\{S\left(\beta_{n_{i}} T\left(f_{n_{i}}\right)+\left(1-\beta_{n_{i}}\right) f_{n_{i}}\right)\right\} \rho$-converges to $S(\beta T(f)+(1-\beta) f)$. Finally, since $\rho$ is uniformly continuous, we get $\left\{\alpha_{n_{i}} S\left(\beta_{n_{i}} T\left(f_{n_{i}}\right)+\left(1-\beta_{n_{i}}\right) f_{n_{i}}\right)+\left(1-\alpha_{n_{i}}\right) f_{n_{i}}\right\}$ $\rho$-converges to $\alpha S(\beta T(f)+(1-\beta) f)+(1-\alpha) f$. The above inequalities imply

$$
\begin{aligned}
\rho(f-h) & =\rho(\alpha S(\beta T(f)+(1-\beta) f)+(1-\alpha) f-h) \\
& =\alpha \rho(S(\beta T(f)+(1-\beta) f)-h)+(1-\alpha) \rho(f-h) \\
& =\alpha \rho(\beta T(f)+(1-\beta) f-h)+(1-\alpha) \rho(f-h) \\
& =\alpha[\beta \rho(T(f)-h)+(1-\beta) \rho(f-h)]+(1-\alpha) \rho(f-h) .
\end{aligned}
$$


Set $r=\rho(f-h)$. Without loss of generality we may assume $r>0$ (otherwise most of the conclusions in the theorem are trivial). Assume that $\liminf _{n \rightarrow \infty} \alpha_{n}>0$. Then $\alpha \neq 0$. Hence

$$
\begin{aligned}
\rho(S(\beta T(f)+(1-\beta) f)-h) & =\rho(\beta T(f)+(1-\beta) f-h) \\
& =\beta \rho(T(f)-h)+(1-\beta) r \\
& =r,
\end{aligned}
$$

which implies $\beta \rho(T(f)-h)=\beta r$. If we assume that $\liminf _{n \rightarrow \infty} \beta_{n}>0$, then $\beta \neq 0$ which implies $\rho(T(f)-h)=r$.

(1) If $\alpha \in(0,1)$ and $\beta>0$, then

$$
\begin{aligned}
\rho(S(\beta T(f)+(1-\beta) f)-h) & =\rho(\alpha S(\beta T(f)+(1-\beta) f)+(1-\alpha) f-h) \\
& =\rho(f-h) .
\end{aligned}
$$

The strict convexity of $\rho$ will imply $S(\beta T(f)+(1-\beta) f)=f$.

(2) If $\alpha \in(0,1)$ and $\beta=0$, then

$$
\rho(f-h)=\rho(S(f)-h)=\rho(\alpha S(f)+(1-\alpha) f-h) .
$$

The strict convexity of $\rho$ will imply $S(f)=f$.

(3) If $\beta \in(0,1)$ and $\alpha>0$, then

$$
\rho(f-h)=\rho(T(f)-h)=\rho(\beta T(f)+(1-\beta) f-h) .
$$

The strict convexity of $\rho$ will imply $T(f)=f$.

(4) If $\alpha, \beta \in(0,1)$, then $T(f)=f$ and $S(\beta T(f)+(1-\beta) f)=f$. Hence $S(f)=f$.

Let us finish the proof of Theorem 2.1. Note that (i) implies $\alpha \in[a, b]$ and $\beta \in[0, b]$. If $\beta=0$, then the conclusion (2) above implies $f \in F(S)$. Otherwise the conclusion (4) will imply $f \in F$. This proves (i).

For (ii), notice that $\alpha \in[a, 1]$ and $\beta \in[a, b]$. Hence the conclusion (3) will imply $f \in F(T)$, which proves (ii).

For (iii), notice that $\alpha, \beta \in[a, b]$. Hence the conclusion (4) will imply $f \in F$. Since

$$
\lim _{n \rightarrow \infty} \rho\left(f_{n}-f\right)=\lim _{n \rightarrow \infty} \rho\left(f_{n_{i}}-f\right)=0
$$

we find that $\left\{f_{n}\right\} \rho$-converges to $f$, which completes the proof of (iii).

If we assume compactness of the domain, Theorem 2.1 will imply the following result.

Theorem 2.2 Assume $\rho \in \Re$ is strictly convex and uniformly continuous. Let $C$ be a nonempty $\rho$-bounded, $\rho$-compact and convex subset of $L_{\rho}$. Let $S, T: C \rightarrow C$ be two nonexpansive mappings. Assume that $F=F(S) \cap F(T) \neq \emptyset$. Fix $f_{1} \in C$. Define $\left\{f_{n}\right\}$ as in (2.1), where $\alpha_{n}, \beta_{n} \in[a, b]$, with $0<a \leq b<1$, and $f_{1}$ is the initial element of the sequence. Then $\left\{f_{n}\right\} \rho$-converges to a common fixed point of $S$ and $T$. 
Proof Since $C$ is $\rho$-compact, $\left\{f_{n}\right\}$ has a $\rho$-convergent subsequence $\left\{f_{n_{i}}\right\}$, i.e., $\left\{f_{n_{i}}\right\} \rho$-converges to $z$. By Theorem 2.1, we have $z \in F$ and $\left\{f_{n}\right\} \rho$-converges to $z$.

The existence of a common fixed point $T$ and $S$ is crucial to the conclusion of Theorems 2.1 and 2.2. Note that each mapping has a nonempty fixed point set. Indeed, let $C$ and $T$ be as in Theorem 2.2. Fix $\varepsilon \in(0,1)$ and $f_{0} \in C$. Define the mapping $T_{\varepsilon}: C \rightarrow C$ by

$$
T_{\varepsilon}(f)=\varepsilon f_{0}+(1-\varepsilon) T(f) .
$$

Then, for any $f, g \in C$, we have

$$
\rho\left(T_{\varepsilon}(f)-T_{\varepsilon}(g)\right) \leq(1-\varepsilon) \rho(f-g) .
$$

Fix $f \in C$. Since is $\rho$-bounded, we get

$$
\rho\left(T_{\varepsilon}^{n+h}(f)-T_{\varepsilon}^{n}(f)\right) \leq(1-\varepsilon)^{n} \rho\left(T_{\varepsilon}^{h}(f)-f\right) \leq(1-\varepsilon)^{n} \delta_{\rho}(C)
$$

for any $n, h \in \mathbb{N}$. Since $C$ is $\rho$-compact, there exists a subsequence $\left\{T_{\varepsilon}^{n_{i}}(f)\right\}$ which is $\rho$-convergent to $h \in C$. We claim that $h$ is a fixed point of $T_{\varepsilon}$. Indeed, since

$$
\rho\left(T_{\varepsilon}^{n_{i}+1}(f)-T_{\varepsilon}(h)\right) \leq(1-\varepsilon) \rho\left(T_{\varepsilon}^{n_{i}}(f)-h\right)
$$

and

$$
\rho\left(T_{\varepsilon}^{n_{i}+1}(f)-T_{\varepsilon}^{n_{i}}(f)\right) \leq(1-\varepsilon)^{n_{i}} \rho\left(T_{\varepsilon}(f)-f\right) \leq(1-\varepsilon)^{n_{i}} \delta_{\rho}(C),
$$

we conclude that $T_{\varepsilon}(h)=h$. Indeed, we have

$$
\rho\left(\frac{h-T_{\varepsilon}(h)}{3}\right) \leq \frac{1}{3}\left(\rho\left(h-T_{\varepsilon}^{n_{i}}(f)\right)+\rho\left(T_{\varepsilon}^{n_{i}}(f)-T_{\varepsilon}^{n_{i}+1}(f)\right)+\rho\left(T_{\varepsilon}^{n_{i}+1}(f)-T_{\varepsilon}(h)\right)\right) .
$$

If we let $n_{i} \rightarrow \infty$, we get $\rho\left(\frac{h-T_{\varepsilon}(h)}{3}\right)=0$, which implies $T_{\varepsilon}(h)=h$. In fact, one can now easily show that $\left\{T_{\varepsilon}^{n}(f)\right\} \rho$-converges to $h$ and that $h$ is independent of $f$ and is the only fixed point of $T_{\varepsilon}$. Clearly,

$$
\rho(T(h)-h)=\rho\left(T(h)-(1-\varepsilon) T(h)-\varepsilon f_{0}\right) \leq \varepsilon \rho\left(T(h)-f_{0}\right) \leq \varepsilon \delta_{\rho}(C) .
$$

Hence $\inf _{f \in C} \rho(T(f)-f)=0$. As $C$ is $\rho$-compact, the fixed point set of $T$ is nonempty. As $\rho$ is strictly convex, $F(T)$ is convex. Clearly $F(T)$ is $\rho$-closed subset of $C$. Hence $F(T)$ is $\rho$-compact. If we assume that $T$ and $S$ commute, i.e., $S \circ T=T \circ S$, then $S(F(T)) \subset F(T)$. Consequently, $S$ and $T$ have a common fixed point. In general, it is not the case that $S$ and $T$ have a common fixed point.

If we take $S=T$ in Theorem 2.2, we get the following result.

Theorem 2.3 [4] Assume $\rho \in \Re$ is strictly convex and uniformly continuous. Let $C$ be a nonempty $\rho$-bounded, $\rho$-compact and convex subset of $L_{\rho}$. Let $T: C \rightarrow C$ be two nonexpansive mapping. Fix $f_{1} \in C$. Define $\left\{f_{n}\right\}$ as in (2.2), where $\alpha_{n}, \beta_{n} \in[a, b]$, with $0<a \leq b<1$, and $f_{1}$ is the initial element of the sequence. Then $\left\{f_{n}\right\} \rho$-converges to a fixed point of $T$. 


\section{Competing interests}

The authors declare that they have no competing interests.

\section{Authors' contributions}

All authors participated in the design of this work and performed equally. All authors read and approved the final manuscript.

\section{Author details}

${ }^{1}$ Department of Mathematics, King Abdulaziz University, Jeddah, 21589, Saudi Arabia. ${ }^{2}$ Department of Mathematical Sciences, University of Texas at El Paso, EI Paso, TX 79968, USA. ${ }^{3}$ Department of Mathematics and Statistics, King Fahd University of Petroleum and Minerals, Dhahran, 31261, Saudi Arabia.

\section{Acknowledgements}

The authors MA Khamsi and AR Khan acknowledge gratefully KACST, Riyad, Saudi Arabia, for supporting Research Project ARP-32-34. The author AAN Abdou gratefully acknowledges the Deanship of Scientific Research at King Abdulaziz University, Jeddah, Saudi Arabia, for supporting this research.

\section{Received: 27 December 2013 Accepted: 7 March 2014 Published: 25 Mar 2014}

\section{References}

1. Luxemburg, WAJ: Banach function spaces. Thesis, Delft (1955)

2. Luxemburg, WAJ, Zaanen, AC: Notes on Banach function spaces I-XII. Proc. Acad. Sci. Amst. A-66, 135-153, 239-263, 496-504, 655-681 (1963); A-64, 101-119 (1964); A-67, 360-376, 493-543 (1964)

3. Nakano, H: Modulared Semi-Ordered Linear Spaces. Maruzen, Tokyo (1950)

4. Bin Dehaish, BA, Kozlowski, WM: Fixed point iteration processes for asymptotic pointwise nonexpansive mapping in modular function spaces. Fixed Point Theory Appl. 2012, 118 (2012). doi:10.1186/1687-1812-2012-118

5. Khamsi, MA, Kozlowski, WM: On asymptotic pointwise contractions in modular function spaces. Nonlinear Anal. 73, 2957-2967 (2010)

6. Khamsi, MA, Kozlowski, WM: On asymptotic pointwise nonexpansive mappings in modular function spaces. J. Math. Anal. Appl. 380, 697-708 (2011)

7. Kozlowski, WM: Advancements in fixed point theory in modular function. Arab. J. Math. (2012) doi:10.1007/s40065-012-0051-0

8. Kozlowski, WM: Notes on modular function spaces I. Comment. Math. 28, 91-104 (1988)

9. Kozlowski, WM: Notes on modular function spaces II. Comment. Math. 28, 105-120 (1988)

10. Kozlowski, WM: Modular Function Spaces. Series of Monographs and Textbooks in Pure and Applied Mathematics, vol. 122. Dekker, New York (1988)

11. Krasnosel'skii, MA, Rutickii, YB: Convex Functions and Orlicz Spaces. Noordhoff, Groningen (1961)

12. Musielak, J: Orlicz Spaces and Modular Spaces. Lecture Notes in Mathematics, vol. 1034. Springer, Berlin (1983)

13. Chen, S: Geometry of Orlicz spaces. Diss. Math. 356, 1-204 (1996)

14. Kaminska, A: On uniform convexity of Orlicz spaces. Indag. Math. 44, $27-36$ (1982)

15. Khamsi, MA, Kozlowski, WM, Reich, S: Fixed point theory in modular function spaces. Nonlinear Anal. 14, 935-953 (1990)

16. Milnes, HW: Convexity of Orlicz spaces. Pac. J. Math. 7, 1451-1486 (1957)

17. Akimovic, BA: On uniformly convex and uniformly smooth Orlicz spaces. Teor. Funkc. Funkc. Anal. Ih Prilož. 15 114-220 (1972) (in Russian)

18. Khamsi, MA, Kozlowski, WM, Shutao, C: Some geometrical properties and fixed point theorems in Orlicz spaces. J. Math. Anal. Appl. 155, 393-412 (1991)

19. Hudzik, H, Kaminska, A, Mastylo, M: Geometric properties of some Calderon-Lozanovskii spaces and Orlicz-Lorentz spaces. Houst. J. Math. 22, 639-663 (1996)

20. Acerbi, E, Mingione, G: Regularity results for a class of functionals with non-standard growth. Arch. Ration. Mech. Anal. 156, 121-140 (2001)

21. Harjulehto, P, Hästö, P, Koskenoja, M, Varonen, S: The Dirichlet energy integral and variable exponent Sobolev spaces with zero boundary values. Potential Anal. 25(3), 205-222 (2006)

22. Heinonen, J, Kilpeläinen, T, Martio, O: Nonlinear Potential Theory of Degenerate Elliptic Equations. Oxford University Press, Oxford (1993)

23. Shanmugalingam, N: Newtonian spaces: an extension of Sobolev spaces to metric measure space. Rev. Mat. Iberoam $16,243-279(2000)$

24. Das, G, Debata, JP: Fixed points of quasinonexpansive mappings. Indian J. Pure Appl. Math. 17, 1263-1269 (1986)

10.1186/1687-1812-2014-74

Cite this article as: Abdou et al.: Convergence of Ishikawa iterates of two mappings in modular function spaces. Fixed Point Theory and Applications 2014, 2014:74 